\title{
Academic resilience as mediator of multidimensional perfectionism and academic performance among gen- $Z$ undergraduate students
}

Owen Z.H. Choo, Kususanto Prihadi

Center of Cyberpsychology and Games, Department of Psychology, HELP University, Malaysia

\begin{tabular}{l}
\hline Article Info \\
\hline Article history: \\
Received Sep 26, 2019 \\
Revised Nov 19, 2019 \\
Accepted Nov 30, 2019
\end{tabular}

Keywords:

Academic performance

Academic resilience

Dimensions of perfectionism

Gen-Z

Undergraduate student

\begin{abstract}
This study focused on the relationship of two dimensions of perfectionism (perfectionistic strivings, and perfectionistic concerns) and academic performance, with the role of academic resilience as mediator. Participants including 132 undergraduate students form age range 18 to 25 , from first year to fifth year in their studies mainly from a Malaysian psychology undergraduate program were asked to fill questionnaires containing measures for perfectionism, academic resilience and academic performance. Although only partial mediations occurred, both hypotheses where academic resilience would mediate relationships between both dimensions of perfectionism and academic performance were supported. Findings suggested that other variables aside academic resilience could have played a role in predicting perfectionist's academic performance. Findings also suggested interplay of academic performance acting as both protective factor and outcome of academic resilience.
\end{abstract}

Copyright (C) 2019 Institute of Advanced Engineering and Science. All rights reserved.

\section{Corresponding Author:}

Kususanto Prihadi,

Department Psychology,

HELP University,

Subang 2 Campus, No.2 Cakerawala Drive, Sect U4, Shah Alam, Selangor, Malaysia.

Email: prihadi.k@help.edu.my

\section{INTRODUCTION}

Perfectionism is a personality trait characterised by the pursuit of flawlessness, setting of excessively high-performance standards, and the tendencies to critically evaluate one's behaviour [1]. Research interests with perfectionism both theoretically and empirically have grown over the years [2]. It has presented itself as an individual differences characteristic worth noticing with strong pervasive implication in domains of mental health [3], and education [4], with various outcomes ranging from motivation, performance, to well-being among students [5, 6]. Rice, Richardson, and Ray [7] summarized that current understanding of perfectionism supports three points. First, perfectionism can be understood as being multidimensional. Second, dimensions of perfectionism can be surmised into two higher-order dimensions, namely perfectionistic strivings (PS), and perfectionistic concerns (PC). Lastly, based on categorization of the two dimensions, perfectionism can be presented as either adaptive (high in PS) or maladaptive (high in PC).

The multifaceted and multidimensional understanding of perfectionism made it seem like a "doubleedged sword" as described by [8]. From the commonly used measurements of perfectionism (Multidimensional Perfectionism Scale Hewitt \& Flett [HFMPS], Frost Multidimensional Perfectionism Scale [FMPS], and Almost Perfect Scale-Revised [APS-R]), two higher-order dimensions can be collectively captured; one being Perfectionistic Strivings and Perfectionistic Concerns [3, 4, 9, 10]. Both PS and PC exert polarizing effect on an individual, with outcomes visible in psychopathology [11], psychological distress [12], health behaviours [13], burnout [4], and academic achievements [3]. PS and PC have proven to be 
handy predictors of cognitive, affective and behavioural outcomes in various domains of work, education and sports [4]. Perfectionism's dual nature on one hand seem to benefit individuals to strive harder to achieve greatness through PS; yet on another hand seem to be individuals' downfall where they are emotionally distress when discrepancy exists between imagined goals and actual result through PC. Hence, this give rise to categorization of perfectionists of either adaptive (high in PS) or maladaptive (high in PC).

Perfectionistic Strivings (PS) is defined as the higher order dimension of perfectionism which individuals hold themselves to very high personal standards and performance while demand nothing less than perfection $[4,9,14]$. When PC is controlled for, PS was found to be adaptive, while only associated with few maladaptive outcomes $[4,15]$. PS is also linked to positive psychological outcomes [8, 16], having better self-esteem, self-efficacy, life satisfaction and psychological well-being [17, 18]. In a professional working setting, having high PS has a positive effect of workaholism, individual achievement, work enjoyment, involvement, and engagement [2]. Having a high standard of performance allows individuals high in PS to employ approaching behaviour in attempt to pursuit of ideal self [9, 19]. They gain performance satisfaction and achievement following their success, where they remain optimistic about future endeavours [19].

Perfectionistic Concerns (PC) is the degree to which individuals are overly self-critical about own performances, excessively concerned about making mistakes, chronic sense of inadequacy in achieving expectations, and negative reaction following unsatisfactory performance $[4,8,14]$. It captures the aspect and subdimension of perfectionism that reflects socially and other-oriented perfectionism (FMPS), worrying about doing wrong (HFMPS), doubts about own performance (HFMPS), and discrepancy between own performance and expectations (APS-R) [15]. Individuals high in PC are marked by their greater sensitivity in perceiving threat, anxiety, and avoidant coping style [4]. They overcritically evaluate themselves, are constantly occupied with other people's expectations and criticism [9]. Therefore, they are prone to more psychological maladjustments, depression, alcoholic tendencies, and perceiving stress [15, 20]. Even if they are successful in achieving their unrealistically high standards, they are unable to achieve satisfaction from it, as they are driven not by the need to achieve, but avoidance coping for negative affect and fear of failure [9, 21]. As described by Lo and Abbott [19], these maladaptive tendencies to perform are ultimately worrying, as it further promotes and perpetuate inadequacy feeling and failure concerns when individuals high in PC faces future struggles.

The other variable of this study is academic resilience. Resilience is the coping of life adversities, in ways that individuals' normal development individuals are not impeded or even [22]. In education's context, academic resilience is the education-specific form of resilience [23, 24], and is defined as the capability to overcome sudden or chronic adversities that negatively disrupt individual's academic development [25]. Even when faced with academic adversities, highly academic resilience individuals would manage to thrive and flourish academically $[23,26]$. Compared sidewise, individuals of higher academic resilience would more likely bounce back and adjust from academic adversities than individuals of lower academic resilience [27]. Perfectionism has been linked with academic resilience; in the face of overbearing stress, perfectionists are often unwilling to admit to imperfections. It was suggested that self-oriented and socially-prescribed perfectionists strive hard to prove themselves to others, so that others view them as equally if not more competent, without flaws and adaptive enough to manage even the toughest scenarios [10]. Over time, this effort to over-strive and maintain a façade is harmful to perfectionist's emotional well-being, which is said to inhibit accumulations of resilience building resources [10].

Despite, when effect of PC is controlled for, PS in its purest form would be adaptive, considering the positive cognitive response to failure [28]. Individuals high in PS are more self-compassionate, more optimistic and less of a pessimist [1], leading towards more protective factor of resilience. This sense of optimism allows individuals of higher PS to better regulate their goals, as they could see the discrepancy in performance as resolvable and this would result in continued effort [29]. Contrary to what Kilbert et al. [10] suggested, Rice, Ray et al. [14] found that adaptive perfectionists (high in PS) are more stress-tolerant. As PS dampened and suppressed the effects of stress, it was suggested that individuals high in PS may be more resilient than others and less likely to get side-railed academically [14]. This finding is also reciprocated by Noh [30] as he found positive relationship between self-oriented perfectionism (a category of adaptive perfectionist high in PS) and academic resilience.

Adaptive perfectionists' approach when facing problems might be the key to why they are welladjusted. Those high in PS would employ a task-oriented coping style, rather than avoidance and escaping [1]. As students of high PS are more mindful of the ever-increasing academic demands, they respond by utilising more problem-focused approaches to the problems, in a way fostering resilience [12]. On the other hand, PC is negatively related to academic resilience. PC has been consistently linked to psychological maladjustments $[8,31]$. Individuals high in PC are more likely to ruminate and be pessimistic, while less associated to be self-compassionate necessary to build up resilience [31]. Their pessimistic and ruminative

Int. J. Eval. \& Res. Educ. Vol. 8, No. 4, December 2019: 637 - 646 
tendencies made them less likely to cognitively reappraise distressing feelings; instead they suppress those feelings [3]. Moreover, these negative emotion tendencies persist especially when their self-worth is under threat from external expectations and criticisms in times of stress [4]. These emotional regulation strategies do not bode well with their emotional well-being, leading to psychological maladjustment.

As Kilbert et al. [10] suggested, socially-oriented perfectionist (a category of maladaptive perfectionist high in PC) is easily paralysed by perceived failure. They perceived themselves as less capable to reduce the discrepancy between actual performance and targeted goal which is important when considering employing a goal-directed action [9]. This perceived failure inhibits socially-oriented perfectionists to utilise existing psychological resources to positively appraise the situation that would promote desire to engage productively to overcome the adversities [10], as they are negatively biased in perceiving lack of resources to reduce the discrepancy [9]. It was further explained that socially-oriented perfectionists are unlikely to engage in resilience building activities such as seeking social support and utilise a diverse pool of effect problem-solving strategies. Instead, they are more likely to engage in catastrophizing and dependency, which are resilience-depleting as focus is shifted from problem-solving to attending emotional needs, through avoidance behaviour [9-10].

Furthermore, academic resilience is perhaps the determining factor in identifying those who will prevail academically and those who will not [24]. Researchers understanding of academic resilience's relationship with academic performance is pretty much in consensus, academic resilience is positively correlated with academic performance [22, 23, 27, 32, 33], even when controlling for risk factors of poverty, family disposition and low socio-economic status [34]. Academic resilience serves as a protective factor for academic performance while at the same time academic performance would feed back to academic resilience as a its protective factor [22]. Highly academically resilience students are more engaged in class, have fewer school related issues, burnout [35]. Highly academically resilient students are also better in self-learning regulation, emotional regulation, and self-regulation [33, 36, 37]. They are academically motivated and possessing higher personal academic standards, while the tenacity to develop skills, capabilities and competency in everyday academic strivings [27], allowing them to be more adaptive in academic setting.

Instead of a passive avoidance coping style, academically resilient students are more able to adopt to prescribed stress [27], given their approach coping style [36]. Academically resilient students see difficulty in studies as challenging while able to see the benefit behind those hardship, allowing them to be more engaged in their academic strivings, while being able to cope with the stress they are facing academically [37]. As academic resilience will reflect a more positive attitude one portrays when facing adverse situation, academic resilience will ultimately translate into academic achievements [27]. All of these provided clear understanding of the positive association between academic resilience and academic performance.

\section{RESEARCH METHOD}

Variables studied were perfectionistic strivings, perfectionistic concerns, academic resilience, and academic performance. Perfectionistic strivings is operationally defined as total score on the High Standards subscale on the Almost Perfect Scale-Revised (APS-R) [38], where a higher total score indicates higher perfectionistic strivings. Perfectionistic concerns is operationally defined as total score on the Discrepancy subscale on the Almost Perfect Scale-Revised [38], where a higher total score indicates higher perfectionistic concerns. Academic resilience is operationally defined as total score on the Academic Resilience Scale (ARS-30 [24]), where a higher total score indicates higher academic resilience. Academic performance is operationally defined as the total score on the Researcher-Generated Questionnaire measuring Cumulative Grade Point Average (CGPA), where a higher score indicates better academic performance.

One hundred and fifty undergraduate students aged between 18 and 25 years of age. Sample size is determined with GPower 3.1 with medium effect size (f2=0.15, a=0.05, power $=0.95$; see Appendix ). Participants were recruited via non-probability convenience and snowball sampling method. Participants were approached and asked to participate on a voluntary basis. Those who are readily available are given a link to the scales in the form of Google Form, they were also asked to distribute the link among their peers. The method was chosen due to limited time frame of the data collection.

The Almost Perfect Scale-Revised (APS-R) was used to measure participants' perfectionistic strivings and perfectionistic concerns. The 24-item scale utilize a 7-point Likert scale ranging from 1 (Strongly Disagree) to 7 (Strongly Agree). The High Standard subscale consist of 7 items (item 1, 5, 8, 12, 14,18 , and 22) which were used to measure perfectionistic strivings, which has strong Cronbach alpha value of .72 [20]. Sample items include "I have high expectations for myself" and "I expect the best from myself". Perfectionistic concerns were measured using the Discrepancy subscale consisting of 12 items (item 3, 6, 9, $11,13,15,16,17,19,20,21$, and 23), yielding high Cronbach alpha value of .91 [20]. Sample items include "I rarely live up to my high standards" and "I am never satisfied with my accomplishments". 
Academic Resilience Scale (ARS-30 [24]) was used to measure participants' academic resilience. The 30-item questionnaire utilize a 5-point Likert scale ranging from 1 "Very Likely" to 5 "Very Unlikely", with high Cronbach alpha reliability of . Participants are given a short vignette which they are asked to imagine themselves as the one experiencing academic adversity that induce significant academic challenge and struggle. Sample items included "I would just give up" and "I would keep trying". Positively phrased items (items 2, 4, 8, 9, 10, 11, 13, 16, 17, 18, 20, 21, 22, 23, 24, 25, 26, 27, 29, and 30) were reversed-scored so that a higher total score will reflect higher academic resilience. A Researcher-Generated Questionnaire was used to measure Cumulative Grade Point Average (CGPA) out of 4.00. Participants were asked to report their current latest CGPA scores. Lastly, a researcher-generated demographic questionnaire was prepared where participants were to detail their age, year of study, major of study and university.

\section{RESULTS AND DISCUSSION}

The aim of this study is to investigate the mediating effect of academic resilience on the relationship of multidimensional perfectionism and academic resilience. Out of the 160 responses collected, 132 responses were taken while 28 responses are removed due to straight lining, not meeting criteria or noncompletion. Respondants' age ranged from 18 to $25(\mathrm{M}=21.33, \mathrm{SD}=1.16)$ and year of study ranged from 1 to $5(\mathrm{M}=2.44, \mathrm{SD}=0.84)$.

Multidimensional perfectionism consists of perfectionistic strivings, and perfectionistic concerns, which are measured using the High Standards subscale and the Discrepancy subscale on the ASP-R [38] respectively. High Standards subscale, measured on a 7-point Likert scale, consisted of 7 items which the total score corresponds to perfectionistic strivings; and Discrepancy subscale, measured on a 7-point Likert scale, consisted of 12 items which scores are totalled to correspond to perfectionistic concerns. Higher scores indicated higher perfectionistic strivings and perfectionistic concerns respectively.

Academic resilience is measured with the totalled score of the 30-items on the ARS-30 [24] with a 5-point Likert scale. Items 2, 4, 8, 9, 10, 11, 13, 16, 17, 18, 20, 21, 22, 23, 24, 25, 26, 27, 29, and 30 are reverse scored. Higher totalled score would indicate higher academic resilience.

\subsection{Mediation assumption testing}

As mediation analysis is derived from multiple regression analysis, assumptions are checked to make sure none were violated. First, assumptions of linearity and homoscedasticity were checked using plot of standardized residuals against standardized predicted values and the partial plots of residuals of outcome variable (academic performance) and each of the predictors (PS, PC, and academic resilience) when both variables are regressed separately on the remaining predictor [39]. Assumptions of linearity and homoscedasticity are met, as all points are randomly and distributed across the plot of standardized predicted values against standardized residuals, and each of the partial plots of academic performance against PS, PC, and academic resilience. It can be safely assumed that PS, PC, and academic resilience all have linear relationship with academic performance, and no heteroscedasticity is present. Furthermore, assumption of multicollinearity was checked from the collinearity statistics in the coefficient table [39]. In a model, the three predictors PS, PC, and academic resilience all had a tolerance statistic above 0.2 and VIF values below 10, which are suggested by Field [39] to be indication of no collinearity in the data. Moreover, assumptions of normality and skewness are not checked as bootstrapping approach is a non-parametric test that does not rely on variables being normally distributed and skewed [20]. Lastly, assumption of independence of observations of predictors were not assessed as there was no reason to assume one when PS and PC are covaried [20].

\subsection{Bootstrap indirect effects mediation analysis}

Using PROCESS Macro v3.1 [40] of SPSS v.24, mediation analyses were conducted using the bootstrapping method. Bootstrapping is used for the indirect effect analysis as it was able to balance validity and power considerations [41]. It was run on the 132 samples with a $95 \%$ bias-corrected confidence interval and 10,000 bootstrap estimates setting (as recommended by Hayes [41]). In the mediation model, perfectionistic strivings and perfectionistic concerns are predictor variables, with academic resilience as the mediator variable, and academic performance as the outcome variable. Perfectionistic strivings and perfectionistic concerns were covaried in the mediation model.

Due to limitation of PROCESS Macro v3.1 [40], the combined indirect effect of the two predictor variables to the outcome variable through the mediator cannot be assessed together. However, PROCESS Macro v3.1 does provide solution to this shortcoming, where researchers can insert the other predictor variable into the model as a covariate. This way, PROCESS Macro can be "tricked" into treating the covariate as the second predictor variable, where the statistical output will be identical as if the model 
included the two predictor variables, with the mediator variable and outcome variable intact [40]. Despite showing the overall model significance, PROCESS Macro are unable to show the overall combined indirect effect for the relationship of the two predictor variables to outcome variable through the mediator. It can only show the indirect effect of one predictor to outcome variable through mediator while controlling for the other predictor variable, one predictor at a time.

The results indicated that perfectionistic strivings and perfectionistic concerns as a model, significantly predicted academic resilience, $\mathrm{F}(2,129)=9.58, \mathrm{p}<.001, \mathrm{R}^{2}=.13$. Perfectionistic strivings significantly predict academic resilience, $\mathrm{b}=1.050, \mathrm{t}(129)=9.11, \mathrm{p}<.001$. Perfectionistic concerns also significantly predict academic resilience, $\mathrm{b}=-.325, \mathrm{t}(129)=-2.81, \mathrm{p}=.006$. The aforementioned results suggested that both perfectionist styles significantly predict academic resilience; nevertheless, perfectionistic concerns contributed negatively, while perfectionistic strivings contributed positively to academic achievements. One-point increment of perfectionistic strivings predicts 1.05 points increment of academic achievements, while one-point increment of perfectionistic concern predicts 0.33 decrease of academic procrastination.

The overall model of perfectionistic strivings, perfectionistic concerns and academic resilience significantly predicted academic performance, $\mathrm{F}(3,128)=8.42, \mathrm{p}<.001, \mathrm{R}^{2}=.16$. Academic resilience significantly predicted academic performance, while controlling for perfectionistic strivings and perfectionistic concerns, $b=-.006, t(128)=-2.91, p=.004$. In other words, without any regard on either of the perfectionistic styles, when academic resilience is increased by a single point, the academic achievements will be reduced by 0.006 points, and despite the decrease seems to be miniscule, the effect is significant. This finding is against most of the previous studies stating that academic resilience contributes positively to academic achievements. This would warrant some thoughts into how academic resilience interacts with other variables in the model to produce such results.

Perfectionistic strivings significantly predicted academic performance, while controlling for perfectionistic concerns and academic resilience, $b=.024, \mathrm{t}(128)=4.02, \mathrm{p}<.001$. Perfectionistic concerns significantly predicted academic performance, while controlling for perfectionistic strivings and academic resilience, $\mathrm{b}=-.010, \mathrm{t}(128)=-4.04, \mathrm{p}<.001$. This finding suggested that students with high PC will develop high academic performance when their PS and academic resilience are taken out of the equation. Nevertheless, the implication of the result is almost impossible to be conducted, because in reality, students will have both PC and PS in their mind, as well as resilience in certain levels, which cannot be taken out from their mindset.

In the total effect model, perfectionistic strivings and perfectionistic concerns as a model, significantly predicts academic performance, $\mathrm{F}(2,129)=7.95, \mathrm{p}<.001, \mathrm{R}^{2}=.11$. Perfectionistic strivings significantly predict academic performance, while only controlling for perfectionistic concerns, $b=.018, t$ $(129)=3.14, p<.002$. Perfectionistic concerns significantly predict academic performance, while only controlling for perfectionistic strivings, $\mathrm{b}=-.009, \mathrm{t}(129)=-3.34, \mathrm{p}=.001$. Higher perfectionistic strivings predicts more academic resilience $(\mathrm{b}=1.050)$, higher perfectionistic concerns predict lesser academic resilience $(b=-.325)$, but academic performance subsequently predicts lower academic performance $(b=-.006)$.

A bias-corrected bootstrapped confidence interval for the indirect effect of perfectionistic strivings on academic performance through academic resilience was significant $(b=-.006)$, based on 10,000 bootstrap samples do not straddle zero, BCa CI [-.0121, -.0017], indicating that mediation likely occurred. However, when controlling for academic resilience and perfectionistic concerns, perfectionistic strivings still predicted academic performance, indicating that a partial mediation occurred. Furthermore, another bias-corrected bootstrapped confidence interval for the indirect effect of perfectionistic concerns on academic performance through academic resilience was significant $(b=.005)$, based on 10,000 samples does not straddle zero, $\mathrm{BCa}$ CI [.0004, .0039], indicating that likely mediation occurred. However, when controlling for academic resilience and perfectionistic strivings, perfectionistic concerns still predicted academic performance, indicating that a partial mediation occurred.

Therefore, the first hypothesis of academic resilience mediates the relationship between perfectionistic strivings and academic performance and second hypothesis of academic resilience mediates the relationship between perfectionistic concerns and academic performance was both supported. However, both mediating effects are only partial. Figure 1 illustrates the summary of the aforementioned results. 


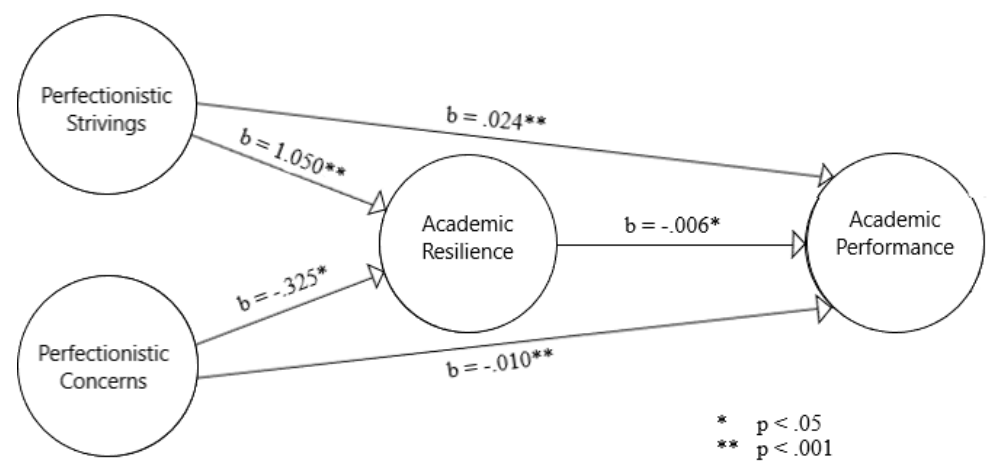

Figure 1. Conceptual diagram for the indirect effect of perfectionistic strivings and perfectionistic concerns on academic performance through academic resilience

\subsection{Mediating role of academic resilience}

Academic resilience did partially mediate the relationship between PS and academic performance, and PC and academic performance. PS was found to positively predict academic resilience, and to positively predict academic performance when controlling for PC, and academic resilience. PC was found to negatively predict academic resilience, and to negatively predict academic performance when controlling for PS, and academic resilience. However, academic resilience was found to be slightly negatively predict academic performance. These results did support the hypotheses that PS and PC would have an indirect effect on academic performance through academic resilience. Individuals high in PS will report higher academic resilience while individuals high in PC will report lower academic resilience, but those high in academic resilience would report slightly lower academic performance while those low in academic resilience would report slightly higher academic performance.

The two higher order perfectionistic dimensions' relationship with academic resilience and academic performance were as predicted and consistent with past research. PS was positively predictive of academic performance, as their approaching behaviour in pursue of excellence [9, 19] which would predict future optimism [19]. The optimism and self-compassion in PS perfectionists [1] also ensured of continued effort in future endeavour [29], a sign of resilience. They are more task-oriented and problem-focused [1, 12]. $\mathrm{PC}$ is consistent with past researches in negatively predicting both academic resilience and academic performance. Their emotional coping when it comes to adversities in life prevents the accumulation of academic resilience and improvements of academic performance [4,19]. PC perfectionists are low in resilience as rumination, pessimism and lack of self-compassion is common among them [31], coupled with the unwillingness to engage in resilience building activities.

On the other hand, the weak negative correlation between academic resilience and academic performance contradicts past researches, when controlling for the shared variances of PS and PC. Recent studies mostly suggested a significant positive relationship between academic resilience and academic performance [32] although some researchers reported weak correlations [22, 27], to the author's knowledge, no past literatures have showed weak negative relationship between academic resilience and academic performance. This would warrant some thoughts into how academic resilience interacts with other variables in the model to produce such results.

\subsection{Academic resilience as a supressor variable and the possible role of stress}

It is speculated that academic resilience exerted a suppression effect upon the relationship between multidimensional perfectionism and academic performance. As described by Field [29], a suppression effect happens when the predictor (academic resilience) is only a significant predictor of an outcome variable (academic performance) when other variables are held constant (PS and PC). The suppressor, when included, increases the predictive validity of another variable [42]. Including academic resilience as a mediator resulted in the direct effect having a greater magnitude of effect than the total effect [42]. As a mediator of PS and academic performance, the indirect effect of academic resilience is negative in magnitude $\left(a_{1} b=-.006\right)$, while the total effect is positive in magnitude $\left(c_{l}=.018\right)$. The indirect effect of academic resilience as mediator of $\mathrm{PC}$ and academic performance is positive in magnitude $\left(a_{2} b=.002\right)$, while the total effect is negative in magnitude $\left(c_{2}=-.009\right)$. Both cases have the indirect effect weaken in magnitude as compared to the total effect, due to the change in sign and decrease in magnitude. 
The apparent suppression effect of academic resilience is only a label that does not suffice as an explanation [40]. Perceived stress emerged as one of the variables that could explain and support the peculiar relationship between these suppressive indirect effects. Considering perceived stress into the picture, Rice et al. [7] claimed that in a group of perfectionists that transitioned across low, moderate, and high stress groups over a period of time, lowly stressed adaptive perfectionist would have the best academic outcome, while highly stressed maladaptive perfectionist would have the worst academic outcome. Interestingly, comparing adaptive perfectionist and maladaptive perfectionist across the moderate stress group; maladaptive perfectionists performed better acadmically than adaptive perfectionist [7]. This would suggest that under certain condition maladaptive perfectionists might actually outperform adaptive perfectionists. In other words, over a period of time of increasing stress, perfectionists (regardless of adaptive or maladaptive perfectionists) would have decreased academic performance, with every transition into the next stress group resulted in near 1.0-point drop of GPA. Generally, as would be expected, lowly stressed adaptive perfectionists performed best academically while highly stressed maladaptive perfectionists performed worst.

Effects of performance decrease began as early as adaptive perfectionist transitioned from the low stress group to the moderate stress group; similarly, the increase of stress across time would also hamper maladaptive perfectionists' academic performance [7]. However, moderately stressed maladaptive perfectionists actually have higher GPAs than adaptive perfectionists of similar stress level, a finding that can be explained as an over-time adaptation in managing stress for maladaptive perfectionists. Due to their sensitivity and tendency to exacerbate minimal stress, maladaptive perfectionists over time adapted to perform under moderate stress, allowing them to manage and mould stress into motivation towards high standards [7]. These motivations are possibly derived as part of avoidance coping [17], in order to avoid aversive consequences of negative evaluation and failure [13, 21].

Tying the findings of the aforementioned previous studies into the context of this current study, perceived stress would be the precursor to academic resilience for PS and PC perfectionists in predicting the indirect effect to academic performance. Academic resilience is the cognitive-affective and behavioural responses of individuals under the academic duress [24]. As adaptive and maladaptive perfectionists are differ in outcome in response to different stress, where adaptive perfectionists are able to buffer against the effects of stress [17] while maladaptive perfectionists exacerbate the effects of stress [7]. Both adaptive perfectionist and maladaptive perfectionist would in this case employ different resilience strategies to deal with adversities reflected in terms of academic resilience.

Under certain stress conditions, maladaptive perfectionists are found to have more favourable outcomes than adaptive perfectionists [7]. Despite individual differences in evaluation of the vignette context, the ARS-30 was still poised to induce considerable stress among respondents as respondents imagine themselves facing academic adversities. Therefore, it should not come as a surprise as the indirect effect of academic resilience would have positive academic outcome for more PC perfectionist, while having negative academic outcome for PS perfectionists. Therefore, it should be expected that without considering perceived stressed of perfectionists (PS or PC, regardless) and only consider academic resilience, one can get situations where maladaptive perfectionists (high in PC) could perform better than adaptive perfectionists (high in PS). To simplify, academic resilience is the outcome of perceived stress, and is only justified if

Despite the potential benefit in deriving motivation in times of heightened stress, PC perfectionists are constantly worrying about their performance neurotically while engaging in dysfunctional coping strategies [17]. The need to maintain a 'perfect' façade of constant top-tier performance is emotionally draining, which could lead to frequent burnout which is also expected among individuals high in PC [4, 17]. It was unknown of the level of stress perceived by the 132 respondents of this study. Nonetheless, taking account of perceived stress of perfectionists could possibly explain how academic resilience suppresses the relationship between the two perfectionism dimensions and academic performance.

\subsection{Partial mediation model}

Another possible reason why academic resilience yielded negative correlation with academic performance when controlling for PS and PC was because of the partial mediation model. In line with explanations above, other variables might also have roles in determining how PS and PC affects academic performance as academic resilience only partially mediated both the model of PS and PC on academic performance. Indeed, the total effect model when PS and PC are predictors explained $11.0 \%$ of the total variance of academic outcome $\left(R^{2}=.11\right.$; see Appendix $\left.\mathrm{J}\right)$. Whereas when academic resilience was added to be included into the multiple regression model as a mediator, the amount of variances explained by the model increased minimally to $16.5 \%\left(R^{2}=.16\right.$; see Appendix J). Academic resilience only accounted for $5.5 \%$ increase in variance explained $\left(R^{2}\right.$ change $=.055, p=.004$; refer to Appendix M).

This reminisced Novotný \& Křeménková's finding [27] where resilience only has a low to moderate effect $(10 \%-24 \%)$ on academic performance. Apparent of the how little variance academic resilience aided 
in explaining the model, perhaps other relevant variables that were overlooked in this study, which could provide a more comprehensive understanding of how multidimensional perfectionism and academic performance interacts. Future researches could consider other variables that could aid in seeing a clearer picture.

\subsection{Limitations and future research}

There were some limitations in this study. First, CGPA might not be the best reflection of academic performance. As with other researchers, this study employs CGPA as measurement of academic performance due to its availability and convenience. However, CGPA is numerical equivalents of grades with increasing numerical symbols assigned to increasing performance of grade. During calculation of GPA, certain data are lost during conversion (e.g. 84 marks is considered 3.75 GPA, but 85 marks is considered 4.00 GPA) where the actual order in ranking might not be preserved

Moreover, the theoretical range of this measurement available is also 'biased' in a way, as students with below 2.00 CGPA would hardly be sampled due to possible academic suspension. In future studies, researchers should look out for more representative measure of academic performance that is easy to use and widely available among students for comparability. It is suggested that instead of academic performance alone, researchers should consider measuring academic success, which includes career success, fulfilling learning outcomes, persistence (academic resilience), attainment of skills and competencies, satisfaction, and academic performance.

Thirdly, causality and temporarily among variables could not be inferred due the cross-sectional study design. Future studies that are interested in studying the suppression effect of academic resilience on performance of perfectionists could benefit from mediation analysis that could do so, with appropriate control and manipulation. The academic resilience recorded in this study could also be skewed. As students progress through their undergraduate programmes, it would only make sense that their past academic experience would in a way help them garner more academic resilience over time. Reports of academic resilience could possibly be negatively skewed as they progressed to their senior years. Without a baseline of comparison from academic resilience during respondents' first year in university, this cannot be said for certain. Although the current study has respondents from various undergraduate programs of local and foreign institutions, 84 out of the 132 (63.6\%) respondents originated from the same psychology undergraduate programme as the author. Further studies could be done by incorporating data from undergraduates from various undergraduate programmes in Malaysia or in the SEA region for a more representative data set.

This study solely focused on residual PS and PC of perfectionism (PS and PC after controlling for the common variance among the two dimensions). As discussed above, the combination of PS and PC would produce meaningful distinctive within-person perfectionism subtypes of adaptive perfectionists, maladaptive perfectionists, and non-perfectionists. Future researches could focus on how each perfectionist subtypes could interact and allow more comprehensive and meaningful view of how PS and PC interplay to in affecting an individual's everyday outcome. This could perhaps provide more comprehensive interventions for perfectionists at risk of unfavourable outcomes

\section{CONCLUSION}

When placed in a model, academic resilience did partially mediate the relationship between PS, PC, and academic performance. PS was found to positively predict academic resilience, and to positively predict academic performance when controlling for PC, and academic resilience. PC was found to negatively predict academic resilience, and to negatively predict academic performance when controlling for PS, and academic resilience. However, academic resilience was found to be slightly negatively predict academic performance. These results did support the hypotheses that PS and PC would have an indirect effect on academic performance through academic resilience. Individuals high in PS will report higher academic resilience while individuals high in PC will report lower academic resilience, but those high in academic resilience would report slightly lower academic performance while those low in academic resilience would report slightly higher academic performance.

While the results generally showed consistence with past literature, pathway b of the model showed contradiction with past studies. When controlling for the variances of PS and PC, academic resilience was showed to slightly negatively predict academic performance. Recent studies mostly suggested a significant positive relationship between academic resilience and academic performance, although some researchers reported weak correlations. While some researchers did report non-significant relationship between the two variables, to the author knowledge, no past literatures have showed peculiar finding of significant weak negative relationship between academic resilience and academic performance. 


\section{REFERENCES}

[1] Y. Luo, Z. Wang, H. Zhang, A. Chen and S. Quan, "The effect of perfectionism on school burnout among adolescence: The mediator of self-esteem and coping style," Personality and Individual Difference, vol. 88(202208), Jan. 2016, doi: 10.1016/j.paid.2015.08.056.

[2] P. Kanten and M. Yesiltas, "The effects of positive and negative perfectionism on work engagement, psychological well-being and emotional exhaustion," Procedia Economics and Finance, 2015, pp. 1367-1375, doi: 10.1016/S2212-5671(15)00522-5.

[3] K. G. Rice, C. M. E. Richardson, and S. Tueller, "The short form of the revised almost perfect scale," Journal of Personality Assessment, vol. 96(3), pp. 368-379, Oct. 2013, doi: 10.1080/00223891.2013.838172.

[4] A. P. Hill \& T. Curran, "Multidimensional perfectionism and burnout: A meta-analysis," Personality and Social Psychology Review, vol. 20(3), Jul. 2015, doi: 10.1177/1088868315596286.

[5] C. L. Noble, J. S. Ashby and P. B. Gnilka, "Multidimensional perfectionism, coping, and depression: Differential prediction of depression symptoms by perfectionism type," Journal of College and Counseling, vol. 17(1), pp. 8094, Apr. 2014, doi: 10.1002/j.2161-1882.2014.00049.x.

[6] J. Stoeber, A. Haskew and C. Scott, "Perfectionism and exam performance: The mediating effect of task-approach goals," Personality and Individual Differences, vol. 74, pp. 171-176, Feb. 2015, doi: 10.1016/j.paid.2014.10.016.

[7] K. G. Rice, C. M. E. Richardson and M. E. Ray, "Perfectionism in academic settings," in Perfectionism, Health and Well-being, F. M. Sirois, and D. S. Molnar, Eds., Switzerland: Springer, 2016, pp. 245-264.

[8] J. Stoeber, and P. Gaudreau, "The advantages of partialling perfectionistic strivings and perfectionistic concerns: Critical issues and recommendations," Personality and Individual Differences, vol. 104, pp. 379-386, Jan. 2017, doi: 10.1016/j.paid.2016.08.039.

[9] F. M. Sirois, D. S. Molnar and J. K. Hirsch, "A meta-analytic and conceptual update on the associations between procrastination and multidimensional perfectionism," European Journal of Personality, vol. 31(2), pp. 137-159, Mar. 2017, doi: 10.1002/per.2098.

[10] J. Kilbert, D. A. Lamis, W. Collins, K. B. Smalley, J. C. Warren, C. T. Yancey, and C. Winterowd, "Resilience mediates the relations between perfectionism and college student distress," Journal of Counseling and Development, vol. 92(1), pp. 75-81, Jan. 2014, doi: 10.1002/j.1556-6676.2014.00132.x.

[11] A. M. Burgess and P. M. DiBartolo, "Anxiety and perfectionism: Relationships, mechanisms, and conditions," in Perfectionism, Health, and Well-being, F. M. Sirois and D. S. Molnar, Eds., Switzerland: Springer International Publishing, 2016, pp. 177-203.

[12] D. M. Dunkley, T. Mandel, and D. Ma, "Perfectionism, neuroticism, and daily stress reactivity and coping effectiveness 6 months and 3 years later," Journal of Counseling Psychology, vol. 61(4), pp. 616-633, Oct. 2014, doi: $10.1037 / \mathrm{cou} 0000036$.

[13] F. M. Sirois, "Perfectionism and health behaviours: A self-regulation resource perspective," in Perfectionism, Health, and Well-being, F. M. Sirois and D. S. Molnar, Eds., Switzerland: Springer, pp. 45-68, 2016.

[14] K. G. Rice, M. E. Ray, D. E. Davis, C. DeBlaere and J. S. Ashby, "Perfectionism and longitudinal patterns of stress for STEM majors: Implications for academic performance," Journal of Counseling Psychology, vol. 62(4), pp. 718-731, Oct. 2015, doi: 10.1037/cou0000097.

[15] M. M. Smith and D. H. Saklofske, "The structure of multidimensional perfectionism: Support for a bifactor model with a dominant general factor," Journal of Personality Assessment, vol. 99(3), pp. 297-303, Aug. 2017, doi: 10.1080/00223891.2016.1208209.

[16] J. Stoeber and P. J. Corr, "A short empirical note on perfectionism and flourishing," Personality and Individual Differences, vol. 90, pp. 50-53, Feb. 2016, doi: 10.1016/j.paid.2015.10.036.

[17] P. B. Gnilka, J. S. Ashby and C. M. Noble, "Adaptive and maladaptive perfectionism as mediators of adult attachment styles and depression, hopelessness, and life satisfaction," Journal of Counseling \& Development, vol. 97(1), pp. 78-86, Jan. 2013, doi: 10.1002/j.1556-6676.2013.00074.x

[18] J. Stoeber, C. R. Davis and J. Townley, "Perfectionism and workaholism in employees: The role of work motivation," Personality and Individual Differences, vol. 55(7), pp. 733-738, Oct. 2013, doi: 10.1016/j.paid.2013.06.001.

[19] A. Lo and M. J. Abbott, "Review of the theoretical, empirical, and clinical status of adaptive and maladaptive perfectionism," Behaviour Change, vol. 32(2), pp. 96-116, Jun. 2013, doi: 10.1017/bec.2013.9.

[20] P. B. Gnilka, S. E. McLaulin, J. S. Ashby, and M. C. Allen, "Coping resources as mediators of multidimensional perfectionism and burnout," Consulting Psychology Journal: Practice and Research, vol. 69(3), pp. 209-222, Apr. 2017, doi: 10.1037/cpb0000078.

[21] F. M. Sirois and D. S. Molnar, "Conceptualizations of perfectionism, health and well-being: An introductory overview," in Perfectionism, Health, and Well-being, F. M. Sirois and D. S. Molnar, Eds., Switzerland: Springer, pp. 45-68, 2016.

[22] J. S. Novotný and L. Křeménková, "The relationship between resilience and academic performance at youth placed at risk," Československá psychologie, vol. 60(6), pp. 553-566, Jan. 2016. [Online]. Available: https://www.researchgate.net/publication/313602814_The_relationship_between_resilience_and_academic_perfor mance_at_youth_placed_at_risk

[23] M. S. Mirza and M. I. Arif, "Fostering academic resilience of students at risk of failure at secondary school level," Journal of Behavioural Sciences, vol. 28(1), pp. 33-50, Jun. 2018. [Online]. Available: http://pu.edu.pk/images/journal/doap/PDF-FILES/03_v28_1_18.pdf

[24] S. Cassidy, "The academic resilience scale (ARS-30): A new multidimensional construct measure," Frontiers in Psychology, vol. 7, pp. 1-11, Nov. 2016, doi: 10.3389/fpsyg.2016.01787. 
[25] A. J. Martin, "Academic bouyancy and academic resilience: Exploring 'everyday' and 'classic' resilience in the face of academic adversity," School Psychology International, vol. 34(5), pp. 488-500, Sep. 2013, doi: $10.1177 / 0143034312472759$

[26] K. Kim and D. T. Hargrove, "Deficient or resilient: A critical review of black male academic success and persistence in higher education," The Journal of Negro Education, vol. 82(3), pp. 300-311, Jun. 2013, doi: 10.7709/jnegroeducation.82.3.0300.

[27] A. Kwek, H. T. Bui, J. Rynne and K. F. S. Kam, "The impacts of self-esteem and resilience on academic performance: An investigation of domestic and international hospitality and tourism undergraduates students," Journal of Hospitality and Tourism Education, vol. 25(3), pp. 110-122, Sep. 2013, doi: 10.1080/10963758.2013.826946.

[28] G. E. Jowett, A. P. Hill, H. K. Hall and T. Curran, "Perfectionism, burnout and engagement in youth sport: The mediating role of basic psychological needs," Psychology of Sport and Exercise, vol. 24, pp. 18-26, May. 2016, doi: 10.1016/j.psychsport.2016.01.001.

[29] K. M. Eddington, "Perfectionism, goal adjustment, and self-regulation: A short term follow-up study of distress and coping," Self and Identity, vol. 13(2), pp. 197-213, Apr. 2013, doi: 10.1080/15298868.2013.781740.

[30] G. O. Noh, "The effects of perfectionism and academic resilience on the level of students' satisfaction with nursing major," The Korean Academic Society of Nursing Education, vol. 23(2), pp. 205-213, May. 2017, doi: 10.5977/jkasne.2017.23.2.205.

[31] M. R. Lizmore, J. G. H. Dunn and J. C. Dunn, "Perfectionistic strivings, perfectionistic concerns, and reactions to poor personal performance among intercollegiate athletes," Psychology of Sport and Exercise, vol. 33, pp. 75-84, Aug. 2017, doi: 10.1016/j.psychsport.2017.07.010

[32] S. M. Debb, D. Colson, D. Hacker and K. Park, "Applying the Connor-Davidson resilience scale for use with third-year African American College Students," The Journal of Negro Education, vol. 97(1), pp. 73-89, Jan. 2018, doi: 10.7709/jnegroeducation.87.1.0073.

[33] E. Hwang and S. Shin, "Characteristics of nursing students with high levels of academic resilience: A crosssectional study," Nurse Education Today, vol. 71, pp. 54-59, Dec. 2018, doi: 10.1016/j.nedt.2018.09.011.

[34] A. Sesma Jr., M. Mannes and P. C. Scales, "Positive adaptation, resilience and the developmental assets framework," in Handbook of Resilience in Children, S. Goldstein and R. B. Brooks, Eds., New York, NY, USA: Springer Science + Business Media, 2013, pp. 427-442.

[35] S. Shin, "The effect of academic stress and the moderating effects of academic resilience on nursing students' depression," The Journal of Korean Academic Society of Nursing Education, vol. 22(1), pp. 14-24, Feb. 2016, doi: 10.5977/jkasne.2016.22.1.14.

[36] A. Abdollahi, P. Carlbring, E. Vaez, S. A. Ghahfarokhi, "Perfectionism and test anxiety among high-school students: The moderating role of academic hardiness," Current Psychology, vol. 37(3), pp.632-639, Sep. 2018, doi: 10.1007/s12144-016-9550-z.

[37] S. Kamstsios and E. Karagiannopoulou, "Exploring relationships between academic hardiness and academic stressors in university undergraduates," Journal of Applied Education and Policy Research, vol. 1(1), pp. 53-73, Jan. 2015. [Online]. Available: https://journals.uncc.edu/jaepr/article/view/317

[38] R. B. Slaney, K. G. Rice, M. Mobley, J. Trippi and J. S. Ashby, "The revised almost perfect scale," Measurement and Evaluation in Counseling and Development, vol. 34(3), pp. 130-145, Oct. 2001, doi: 10.1080/07481756.2002.12069030.

[39] A. Field, Discovering Statistics using IBM SPSS Statistics, 4th ed. London: Sage, 2013.

[40] A. F. Hayes, Introduction to Mediation, Moderation, and Conditional Process Analysis: A Regression-based Approach, 2nd ed. New York, NY: Guildford Press, 2018.

[41] A. F. Hayes, Introduction to Mediation, Moderation, and Conditional Process Analysis: A Regression-based Approach, New York, NY: Guildford Press, 2013.

[42] Rucker, D. D., Preacher, K. J., Tormala, Z. L., \& Petty, R. E., "Mediation analysis in social psychology: Current practices and new recommendations," Social and Personality Psychology Compass, vol. 5(6), pp. 359-371, 2011. 